\title{
Acute abdomen in a septuagenarian
}

\author{
Biggs Saravanan Ramachandran • Mohammed Ali • \\ Anbalagan Amuthan
}

Published online: 25 May 2012

(C) Indian Society of Gastroenterology 2012

A 72-year-old lady presented with abdominal pain and distention of 3 days duration. She did not pass flatus and stools and had few episodes of bilious vomiting for 2 days. She had no past history of abdominal pain or surgery. Coronal CT scan section of abdomen (Fig. 1) showed classical Rigler's triad of pneumobilia, dilatation of small bowel loops and ectopic gallstone which is characteristic of gallstone ileus [1]. At surgery, the stone was $6 \mathrm{~cm} \times 4 \mathrm{~cm}$ in size without any facets. The obstruction was at the midjejunal level.

Often gallstones causing gallstone ileus enter the bowel through cholecystoduodenal fistula.

Management is mainly surgical enterolithotomy. During surgery the finding of facets in the retrieved gallstones may indicate presence of other stones. Due to the emergent nature, patient's old age and the complex mass involving the gallbladder and the cholecystoduodenal fistula cholecystectomy and fistula repair can be done at a later date once the patient recovers. Nearly $17 \%$ of them present with recurrent gallstone ileus or other biliary complications [2]. Hence interval cholecystectomy and fistula repair is prudent. Other treatment option is enteroscopic removal.

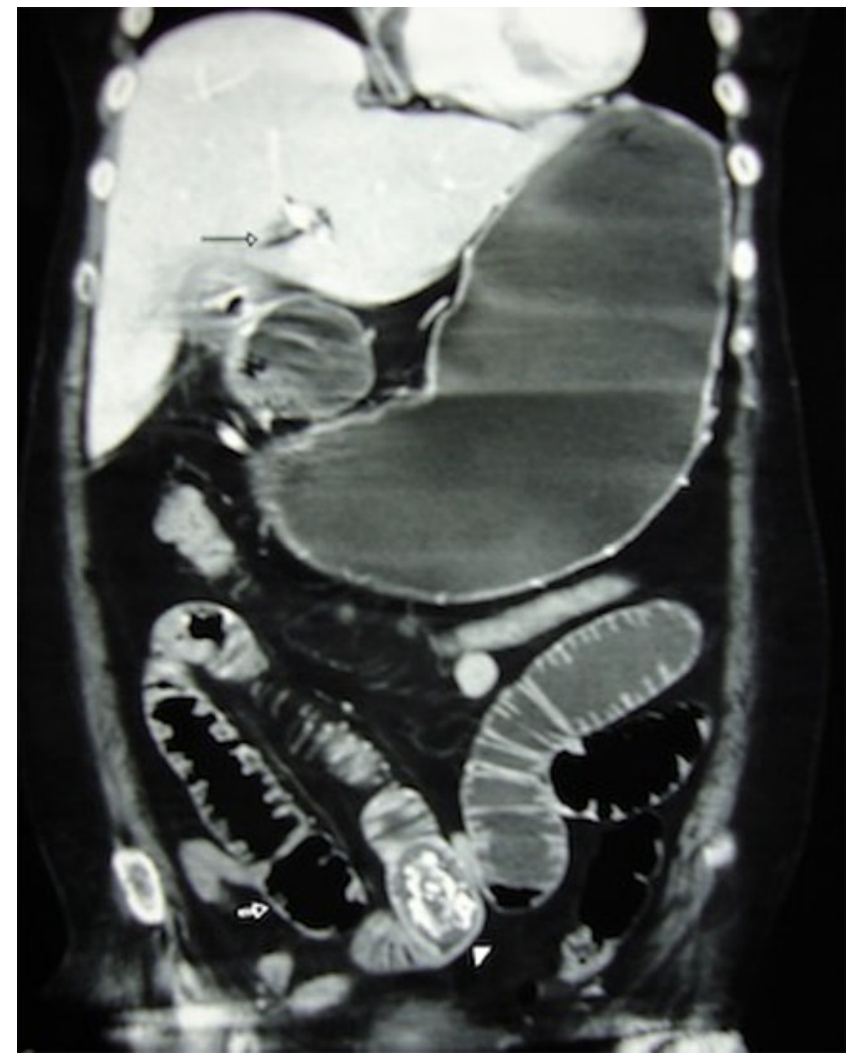

Fig. 1 Shows the classical Rigler's triad. Pneumobilia (black arrow), dilated small intestinal loops (white arrow) and ectopic gallstone in small intestine (white arrowhead)

\section{References}

1. Rigler LG, Borman CN, Noble JF. Gallstone obstruction: pathogenesis and roentgen manifestation. JAMA. 1941;117:1753-9.

2. Reisner RM, Cohen JR. Gallstone ileus: a review of 1001 reported cases. Am Surg. 1994;60:441-6. 